\title{
Serodiagnosis of Tuberculosis in Asian Elephants (Elephas maximus) in Southern India: A Latent Class Analysis
}

\author{
Shalu Verma-Kumar ${ }^{1,29}$, David Abraham ${ }^{39}$, Nandini Dendukuri ${ }^{4,5}$, Jacob Varghese Cheeran ${ }^{6}$, \\ Raman Sukumar ${ }^{2}$, Kithiganahalli Narayanaswamy Balaji ${ }^{1 * 9}$
}

1 Department of Microbiology and Cell Biology, Indian Institute of Science, Bangalore, Karnataka, India, 2 Centre for Ecological Sciences, Indian Institute of Science, Bangalore, Karnataka, India, 3 Asian Nature Conservation Foundation, Bangalore, Karnataka, India, 4 Department of Medicine, McGill University, Montreal, Quebec, Canada, 5 Department of Epidemiology, Biostatistics and Occupational Health, McGill University, Montreal, Quebec, Canada, 6 Cheerans Lab (P) Limited, Thrissur, Kerala, India

\begin{abstract}
Background: Mycobacterium tuberculosis, a causative agent of chronic tuberculosis disease, is widespread among some animal species too. There is paucity of information on the distribution, prevalence and true disease status of tuberculosis in Asian elephants (Elephas maximus). The aim of this study was to estimate the sensitivity and specificity of serological tests to diagnose $M$. tuberculosis infection in captive elephants in southern India while simultaneously estimating sero-prevalence.

Methodology/Principal Findings: Health assessment of 600 elephants was carried out and their sera screened with a commercially available rapid serum test. Trunk wash culture of select rapid serum test positive animals yielded no animal positive for M. tuberculosis isolation. Under Indian field conditions where the true disease status is unknown, we used a latent class model to estimate the diagnostic characteristics of an existing (rapid serum test) and new (four in-house ELISA) tests. One hundred and seventy nine sera were randomly selected for screening in the five tests. Diagnostic sensitivities of the four ELISAs were $91.3-97.6 \%$ (95\% Credible Interval (CI): $74.8-99.9)$ and diagnostic specificity were $89.6-98.5 \%$ (95\% Cl: 79.4-99.9) based on the model we assumed. We estimate that 53.6\% (95\% Cl: 44.6-62.8) of the samples tested were free from infection with M. tuberculosis and $15.9 \%(97.5 \% \mathrm{Cl}: 9.8$ - to 24.0$)$ tested positive on all five tests.

Conclusions/Significance: Our results provide evidence for high prevalence of asymptomatic M. tuberculosis infection in Asian elephants in a captive Indian setting. Further validation of these tests would be important in formulating area-specific effective surveillance and control measures.
\end{abstract}

Citation: Verma-Kumar S, Abraham D, Dendukuri N, Cheeran JV, Sukumar R, et al. (2012) Serodiagnosis of Tuberculosis in Asian Elephants (Elephas maximus) in Southern India: A Latent Class Analysis. PLoS ONE 7(11): e49548. doi:10.1371/journal.pone.0049548

Editor: Jagadeesh Bayry, Institut National de la Santé et de la Recherche Médicale U 872, France

Received July 12, 2012; Accepted October 10, 2012; Published November 16, 2012

Copyright: (C) 2012 Verma-Kumar et al. This is an open-access article distributed under the terms of the Creative Commons Attribution License, which permits unrestricted use, distribution, and reproduction in any medium, provided the original author and source are credited.

Funding: The study was funded by the Department of Science and Technology, Government of India by award of grant SR/WOS-A/LS-128/2009 to Dr. Shalu Verma-Kumar. The funders had no role in study design, data collection and analysis, decision to publish, or preparation of the manuscript.

Competing Interests: Jacob Varghese Cheeran is employed by Cheerans Lab (P) Limited. This does not alter the authors' adherence to all the PLOS ONE policies on sharing data and materials. Cheerans Lab (P) Limited had no role in developing the serological tests described in the current investigation.

*E-mail: balaji@mcbl.iisc.ernet.in

9 These authors contributed equally to this work.

\section{Introduction}

Conservation medicine enables us to rethink the linkages between human, animal, and environmental health [1], [2]. As wildlife populations become more fragmented and less genetically diverse stochastic events leading to disease outbreaks could become more common. A case in hand is that of the Asian elephant (Elephas maximus) an "Endangered" flagship species and featuring on the '2010 IUCN Red List of Threatened Species' [3]. About 39,463-47,427 wild elephants are found in their 13 range countries in Asia [4]. Estimates of Asian elephant numbers in the wild in India are 26,000-28,000 of which 14,000 are found in southern India [5]. About 3467-3667 elephants are also held in captivity in India (http://envfor.nic.in/pe/PE\%20Note.pdf) at forest camps, temples, zoological gardens and circuses, thus constituting a substantial population living in close proximity to humans.

Both humans and elephants are susceptible to infection primarily with $M$. tuberculosis. Importantly, TB is a serious zoonotic disease in elephants [6], [7], [8], [9], [10] and infects about 11$25 \%$ of tested captive elephant populations in USA, India and Nepal [11]. The transmission, pathobiology and immune correlates of TB are poorly understood in Asian elephants. There is paucity of information on the time intervals between exposure, seroconversion, and shedding of the bacilli as also latent versus active disease status. Equivalent to the culture of human sputum [12], [13], [14] trunk wash culture for isolation of M. tuberculosis remains the 'gold standard' of ante-mortem TB diagnostics in elephants [15]. In its absence, ante-mortem TB diagnosis presents a conundrum [16]. Glinical signs such as chronic weight loss, weakness, anorexia, exercise intolerance and abnormal discharge 
from the trunk [17] are frequently absent or seen at the terminal stages. Intradermal tuberculin test [17], [18] and radiographic thoracic evaluation [17] are unsuccessful in elephants. The GenProbe Amplified Mycobacterium tuberculosis Direct Test (MTD; Gen-Probe, San Diego, CA, USA) has found limited use [19], while ELISA [17], [20], restriction fragment length polymorphism (RFLP) [6], [17], [18] and serological tests such as the dual path platform (VetTB test), multi-antigen print immunoassay (MAPIA) and the rapid serum test (RT, ElephantTB STAT-PAK) from Chembio Diagnostics (Medford, USA) have also been evaluated in elephants [21], [22], [23].

In this study, we report screening of elephants with RT and four in-house ELISAs using $M$. tuberculosis $\mathrm{H} 37 \mathrm{Rv}$ antigens EsxA-6 kDa early secretory antigenic target (ESAT-6) (Rv3875); EsxB-10 kDa culture filtrate antigen (CFP10) (Rv3874); PE_PGRS17 (Rv0978c) and PE_PGRS11 (Rv0754). The ESAT-6 and CFP10 proteins function in inducing interferon gamma $(\mathrm{IFN} \gamma)$ from memory effector cells upon infection with pathogenic mycobacteria [24], [25]. The proline glutamic acid (PE) and proline-proline-glutamic acid (PPE) families of acidic, glycine-rich proteins are unique to the Mycobacteria [26] and many function as cell surface antigens. The PE_PGRS11 (Rv0754) is a hypoxia responsive gene that encodes a functional phosphoglycerate mutase [27] and PE_PGRS17 and PE_PGRS11 antigens induce maturation and activation of human dendritic cells [28].

Performance characteristics of a diagnostic test should ideally enable us to distinguish between infected and non-infected animals. Notably, estimation of DSe (the proportion of infected animals correctly identified by the diagnostic test) and DSp (the proportion of non-infected animals accurately identified by the diagnostic test) of any index test is generally derived by comparing it to a standardized and validated 'gold standard' with the assumed sensitivity and specificity of $100 \%$ [29], [30], [31]. However, the gold standard could suffer from serious deficiencies. For example, the ante-mortem trunk wash culture for $M$. tuberculosis/M. bovis in Asian elephants is reported to suffer from poor sensitivity [17], [22], logistical issues in sample collection and processing and slow turnaround time. In view of these observations, we have utilized Latent Class Analysis (LCA) of five imperfect serological tests to estimate and derive the probability of $M$. tuberculosis infection in elephants.

\section{Results}

\section{Sampling in Elephants}

About 600 captive Asian elephants were visited for health assessment in the three southern Indian states of Kerala, Karnataka and Tamil Nadu. The sampling included healthy individuals as well as animals with alternative diagnosis such as chronic arthritis, impaction, and other non-specific symptoms such as anemia and emaciation. Sera from 179 animals were randomly selected for this study. Trunk-wash culture for isolation of $M$. tuberculosis in select RT positive elephants was carried out with no elephant testing positive. Post-mortem examination of one RT positive elephant revealed lung nodules from which $M$. tuberculosis was cultured. Ante-mortem serum from this animal was used as positive control in standardizing the ELISAs and immunoblot assay.

\section{Evaluation of Humoral Immunoreactivity}

One hundred and seventy nine elephants were screened for differential B-cell responses using $\mathrm{RT}$ and recombinant $M$. tuberculosis $\mathrm{H} 37 \mathrm{Rv}$ antigens in ELISA format. Only selected reference samples were tested by immunoblot analysis using the four recombinant antigens and the results were not included in the LCA model. The immunoblots (Figure 1) were not quantitative in nature. The RT readout gave either a positive or negative test result (binary outcome), while the ELISA results were continuous numerical outcome values (continuous outcome). We dichotomized each continuous test result using a Weibull mixture model that assumed the elephants were a mixture of two latent groups those with the antigen and those without. The cut-off value was the point of intersection of the two Weibull distributions. The cutoff values were 0.2 for ESAT-6, 0.337 for CFP10 and 0.22 for both PE_PGRS11 and PE_PGRS17. Test value greater than each cut-off was deemed to be a positive test. Of the 179 elephants 33 tested positive in RT, 37 in ESAT-6 ELISA, 41 in CFP10 ELISA, 64 in PE_PGRS11 ELISA and 78 in PE_PGRS17 ELISA (Figure 1).

\section{Analyzing Diagnostic Test Results using LCA}

The LCA assumed in Figure 2 has sixteen latent classes. The sero-prevalence associated with each latent class is listed in Table 1. In the absence of any prior information, we chose to use a 'noninformative' prior distribution for the prevalence of $M$. tuberculosis infection, allowing for equal weight of all values from $0 \%$ to $100 \%$. Using the posterior distribution, we report that $53.6 \%$ (97.5\% CI: $44.6 \%$ to $62.8 \%$ ) of the sera samples we tested did not carry any of the $M$. tuberculosis antibodies measured by the 5 tests. This estimate is higher than the 15\% sero-prevalence reported by Abraham et al. (2008, Report submitted to Project Elephant, Ministry of Environment and Forests, Government of India) using RT in the same population of captive elephants. We report that the percentage of $M$. tuberculosis infected animals testing positive in all five tests is $15.9 \%$ (97.5\% CI: 9.8\%-24.0\%). The DSe and DSp of each test with respect to the target antibody that it is designed to detect is listed in Table 2. Thus the PE_PGRS11 ELISA had the highest DSe of $97.6 \%(97.5 \%$ CI: $88.6 \%-99.8 \%)$ and DSp of 98.5\% (97.5\% CI: 93.6\% - 99.9\%). Table 2 also lists the DSe and DSp of each test in detecting the presence of at least one antibody (Table 1 lists 15 latent classes that are positive for at least one antibody) as well as the latent antibodies it is not designed to detect. The main difference between our model and other latent class models is that we recognize that the different tests are measuring different latent variables. Thus, for example, we are able to comment not only on how well RT measures the antigens it is supposed to detect but also on how well it measures $M$. tuberculosis infection that is picked up by other antigens. Finally, the observed and predicted numbers of elephants for each combination of test results (Table 3) agree quite well suggesting that the model fits the data adequately.

\section{Discussion}

Latent class analysis is used in a scenario wherein the gold standard assessment of disease is unavailable and the true infection status unknown but the results of multiple imperfect tests are known [32], [33], [34]. This analysis is attractive because it does not arbitrarily treat one of the tests as a perfect gold-standard with $100 \%$ sensitivity and specificity. It allows intuitive construing of data for input and helps in understanding the uncertainties associated with the predicted prevalence estimates. A large number of reports using LCA in veterinary diagnostic tests have been published [35], [36], [37], [38], [39], [40]. This study uses LCA to estimate the diagnostic test characteristics of five serological tests and is the first report of this analysis used to study TB infection in elephants. This model recognizes that each 

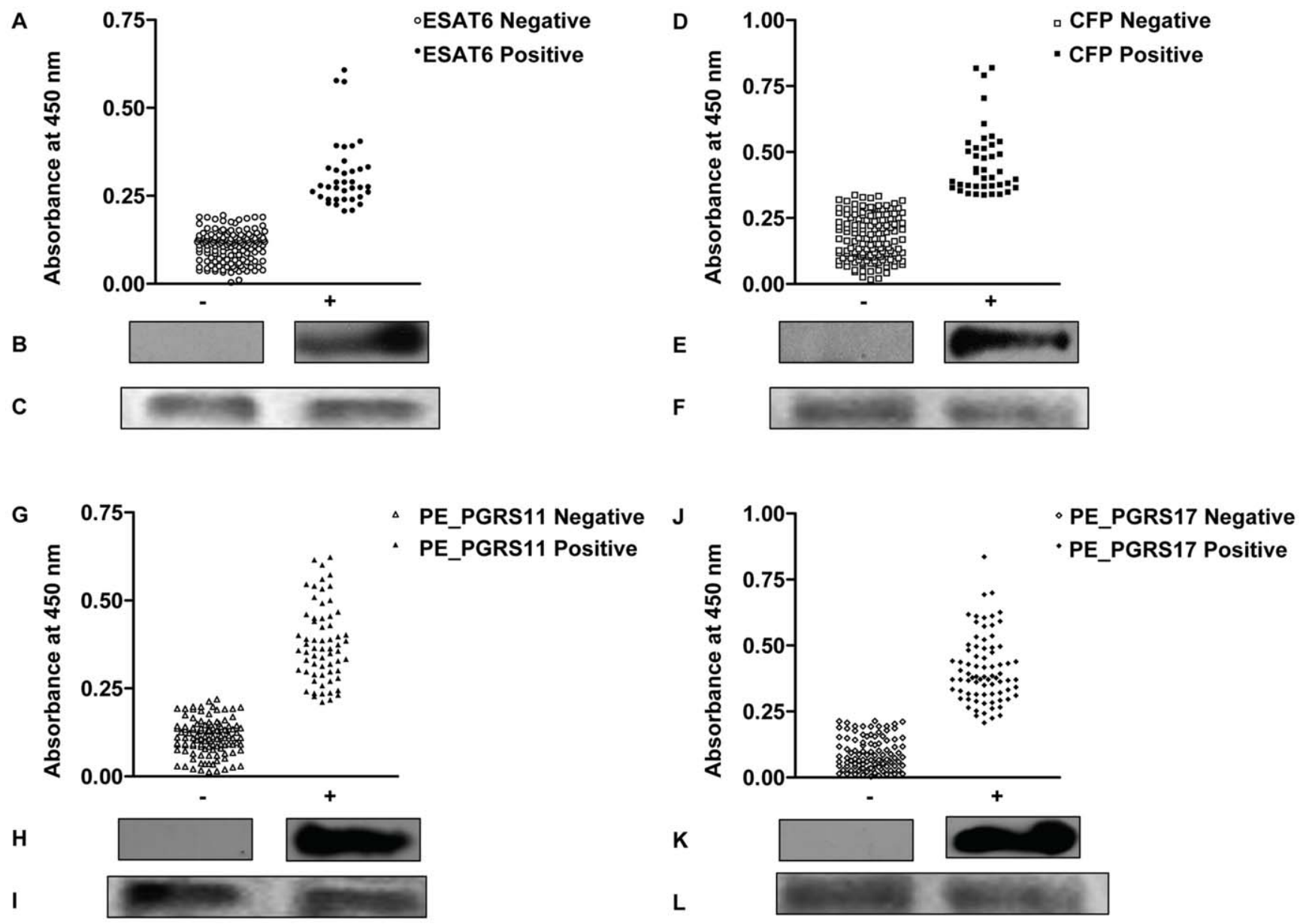

Figure 1. Differential humoral reactivity of four mycobacterial antigens with Asian elephant sera. For ELISA, elephant sera (1:200) was allowed to react with ESAT-6 $(1 \mu \mathrm{g} / \mathrm{ml})($ A), CFP10 $(0.5 \mu \mathrm{g} / \mathrm{ml})(\mathrm{D})$, PE_PGRS11 $(0.25 \mu \mathrm{g} / \mathrm{ml})(\mathrm{G})$ and PE_PGRS17 $(0.25 \mu \mathrm{g} / \mathrm{ml})(\mathrm{J})$. Scatter plots show the total number of animals testing seronegative and seropositive for each antigen. For immunoblotting, $10 \mu \mathrm{g}$ of each transferred protein ESAT-6 (C), CFP10 (F), PE_PGRS11 (I) and PE_PGRS17 (L) was first stained with Ponceau to check for loading control. Next, individual lanes were cut out of the blot and probed with sera from reference negative and positive animals. $B, E, H, K$ represent immunoblots for one representative negative and positive animal each. The westerns were not quantitative in nature.

doi:10.1371/journal.pone.0049548.g001

test is measuring a different target latent variable, which is in turn associated with the presence of $M$. tuberculosis infection.

Test validation in the absence of suitable reference samples is extremely challenging [30], [31]. Culture for clinical isolation of M. tuberculosis remains the 'gold standard' of diagnostics; however a positive culture result is more likely in animals with advanced stage of disease. Validation of DSe based on positive culture results may result in its overestimation under field conditions [38], [40] and DSe may also vary with severity of disease. Validation of DSp entails testing of individuals and herds free from infection (a condition unlikely in TB endemic countries) [41] and may not be constant across different populations [38], [40]. Variables such as methods and their operational characteristics, expertise of the diagnostician, variations in host-pathogen interactions and difference in true disease prevalence rates may contribute to changes in performance of tests. Thus, test performance characteristics would need careful re-evaluation when used in different settings.

In order to decide the cut-off for our new in-house ELISAs, we had the choice of using either the RT as an imperfect gold standard or a mixture model. Even though the RT and ELISA work on the same biological principle, we did not use the former approach as the RT does not contain any PE_PGRS antigens.
The mixture model for continuous data has been used for tuberculin skin test induration data among other applications [42]. The LCA is based on the premise that the true disease status is a common latent (or unobserved) variable associated with several imperfect tests that measure the same disease [43]. For the LCA, we delineated 16 latent classes which are the different combinations of the antibodies detected in the study. It would be interesting to link the latent classes to other disease parameters such as mortality and clinical signs. The disadvantage of using the LCA model is that it dichotomizes test results and thus does not use all the information derived from continuous test results. The choice of model used is dictated by the type of data and whether the model assumptions are satisfied. The model we propose is complex and subject to our interpretation. However, it incorporates results from five serological assays independent of culture results, estimates the true seroprevalence within the sampled population and the true disease status of each animal sampled therein.

Only about $10 \%$ of the $M$. tuberculosis proteome generates human antibody responses, and this immunoproteome contains predominantly membrane-associated and secreted proteins [44]. Differences in antibody profiles seen in TB patients need to factor in host characteristics, bacillary burden and metabolic state and 


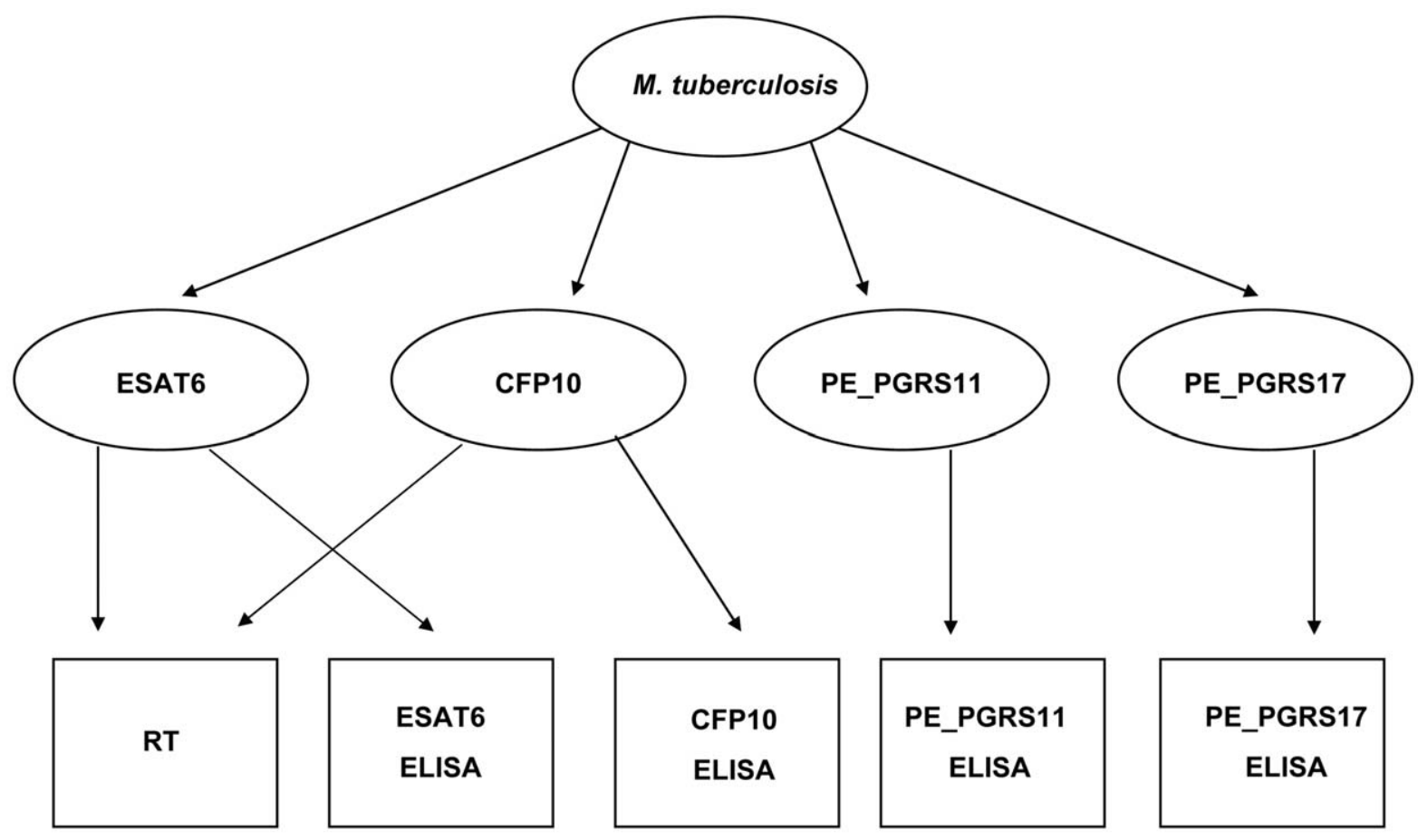

Figure 2. Schematic for the multiple latent variable model used in this study. The parameters to be estimated are depicted in oval shapes and the observed diagnostic test results are represented in rectangular shapes. While RT contains both the ESAT- 6 and CFP10 antigens, the ELISAs are specific for one antigen each.

doi:10.1371/journal.pone.0049548.g002

protein expression by the infecting strain of M. tuberculosis [44], [45]. Additional complexity is introduced by the multiple clinical manifestations of disease in humans [46]. This host and pathogen derived heterogeneity has led to several reviews highlighting the shortcomings of TB immunodiagnostics [47], [48], [49], [50].

Circulating antibodies have been evaluated as biomarkers for TB since 1898 [51]. As compared to other members of the PE family (including PG_PGRS) or mycobacterial antigens, PE_PGR11 and PE_PGR17 have been shown to elicit stronger and differential antibody response in humans. Our laboratory has previously reported that PE_PGR11 and PE_PGR17 elicited antibodies in adult humans with active pulmonary infection, and in child patients with pulmonary or extrapulmonary TB [27], [28], [52]. Serology studies have demonstrated that antibodies reactive with a recombinant carboxyterminal fragment of the PE_PGRS protein from Rv1759c [53] or with the PGRS domain of Rv3367 [54] are present in human sera of patients infected with TB. The ESX protein family (for example, Rv3881c and Rv3784) are preferentially recognized antibody targets in active $\mathrm{TB}$ in humans [44]. The proteins CFP10 (Rv3784) and ESAT6 (Rv3785) have been evaluated in a number of veterinary serological assays [21], [22], [55], [56]. This approach is now giving way to whole proteome screening to identify $\mathrm{TB}$ associated proteins and the dynamics of antibody response they elicit during disease.

The past decade has seen decline of Asian elephant populations in most range countries with the exception of India and Sri Lanka [4]. Population depletion reduces the risk of host-specific infectious diseases except when the pathogen resides in reservoir hosts or when captivity results in increased infectious disease transmission [57]. Thus, conservation strategies that increase population density or cross-species contact such as in zoos, reserves and other captive conditions need careful evaluation in light of the risks of such infection. Cases of cross-species TB outbreaks in zoos are well documented [58]. The management of $\mathrm{TB}$ in Asian elephants in India remains inadequate owing to a number of issues including historical and cultural context of captivity of the species, legality of ownership (government owned vs. privately- owned) or the costs associated with the treatment. Albeit the United States Department of Agriculture has drafted clear guidelines [15] current need involves a creation of guidelines in regard to regulation of $\mathrm{TB}$ infections in elephants in India. Thus, our current study clearly proposes periodic verification/testing for TB in captive elephants as well as in-contact personnel.

Factors governing the initiation and expansion of ensuing immunity to wide ranging infections in elephants are not well understood. Further, detailed analysis of various effector roles played by the key components of immune systems such as $\mathrm{T}$ cells, $\mathrm{B}$ cells, macrophages or dendritic cells, complement and cytokines requires extensive investigation. For example, presence of five subclasses of IgG has been demonstrated in African elephant (Loxodonta africana) [59], [60]. Further, the genomic organization of the $\operatorname{IgH}$, Igא, and Ig $\lambda$ loci of the African elephant has been identified [61], [62]. Immunoreactivity analysis demonstrated the role for complement and antibodies during infections with African horse sickness [63], [64] Bluetongue [63] and Mycoplasmosis [65]. Interestingly, Alpha Napthyl Acetate Esterase activity was utilized as a $\mathrm{T}$ cell marker to demonstrate $\mathrm{T}$ lymphocyte distribution in peripheral blood [66] and presence of functional CD genes in the African elephant [67]. Not much tuberculosis disease stage-specific information is available in Asian elephants and vaccination with $M$. bovis BCG has not been evaluated in elephants. Lyaschenko et al. [21] reported that Multiantigen print immunoassay (MAPIA) and RT could pick up serum IgG to ESAT-6 and other proteins up to 3.5 years and 4.0 years respectively prior to culture of $M$. 
Table 1. Defining the sixteen latent classes and calculating their sero-prevalence rates.

\begin{tabular}{|c|c|c|c|}
\hline \multirow[t]{2}{*}{ Class No. } & \multirow{2}{*}{ Latent class definition } & \multicolumn{2}{|l|}{ Seroprevalence } \\
\hline & & $\begin{array}{l}\text { Median }(95 \% \\
\text { CI)* }\end{array}$ & Mean \\
\hline Class 1 & Mtb $^{* *}+$, All antibodies + & $15.9(9.8,24.0)$ & 16.2 \\
\hline Class 2 & Mtb+, ESAT6+, CFP10+, PE-PGRS11+ & $1.3(0.1,4.3)$ & 1.5 \\
\hline Class 3 & Mtb+, ESAT6+, CFP10+, PE-PGRS17+ & $0.4(0.0,2.1)$ & 0.6 \\
\hline Class 4 & $\begin{array}{l}\text { Mtb+, ESAT6+, PE-PGRS11+, PE- } \\
\text { PGRS17+ }\end{array}$ & $0.8(0.0,4.4)$ & 1.2 \\
\hline Class 5 & $\begin{array}{l}\text { Mtb+, CFP10+, PE-PGRS11+, PE- } \\
\text { PGRS17+ }\end{array}$ & $3.1(0.2,10.3)$ & 3.7 \\
\hline Class 6 & Mtb+, ESAT6+, CFP10+ & $0.8(0.0,3.2)$ & 1.0 \\
\hline Class 7 & Mtb+, ESAT6+, PE-PGRS11+ & $0.6(0.0,3.2)$ & 0.9 \\
\hline Class 8 & Mtb+, ESAT6+, PE-PGRS17+ & $0.5(0.0,2.3)$ & 0.7 \\
\hline Class 9 & $\mathrm{Mtb}+$, CFP10+, PE-PGRS11+ & $0.5(0.0,2.6)$ & 0.7 \\
\hline Class 10 & Mtb+, CFP10+, PE-PGRS17+ & $0.4(0.0,2.3)$ & 0.6 \\
\hline Class 11 & Mtb+, PE-PGRS11+, PE-PGRS17+ & $11.9(4.2,18.5)$ & 11.7 \\
\hline Class 12 & Mtb+, ESAT6+ & $0.7(0.0,3.5)$ & 1.0 \\
\hline Class 13 & Mtb+, CFP10+ & $0.5(0.0,2.6)$ & 0.7 \\
\hline Class 14 & Mtb+, PE-PGRS11+ & $0.7(0.0,3.1)$ & 0.9 \\
\hline Class 15 & Mtb+, PE-PGRS17+ & $4.8(0.2,11.8)$ & 5.1 \\
\hline Class 16 & Mtb-, All antibodies- & $53.6(44.6,62.8)$ & 53.6 \\
\hline
\end{tabular}

Latent class definition and the seroprevalence rate for each class thus defined in the LCA model for studying TB in Asian elephants in southern India. The latent classes are different combinations of the latent variables (i.e. true presence of the antibodies) which are present if $M$. tuberculosis infection is present. * Median estimate is at the $50 \%$ quantile while the $2.5 \%$ and $97.5 \%$ quantiles define a $95 \%$ credible interval $(\mathrm{Cl}) .{ }^{* *} M$. tuberculosis infection.

doi:10.1371/journal.pone.0049548.t001

tuberculosis from trunk washes. They reported that ESAT6 and CFP10 were the immunodominant antigens elicited upon infection of elephants with M. tuberculosis/M. bovis [21], [23]. Significantly, in addition to ESAT-6 and CFP-10, we report high DSe and DSp for the two PE_PGRS ELISAs based on a latent class model that recognizes that the different tests are designed to measure different antigens. The DSe and DSp for the ELISAs we developed for ESAT6 and CFP10 were comparable to the commercially available RT test. Importantly, we have attempted to address immunoreactive potential as well as serodiagnostic utility of selected antigens of $M$. tuberculosis $\mathrm{H} 37 \mathrm{Rv}$ which would help in our understanding of the pathophysiological attributes of TB infection in elephants.

Serology remains an attractive first step in TB diagnosis in wildlife. It is simple, quick, affordable and does not require repeated handling of animals. Once elicited, the antibody response is sustained while the trunk wash culture may yield intermittent results in elephants. Identifying serological correlates of active TB in elephants and their use in antitubercular treatment monitoring [21], [23], [68] could be potentially useful tools in situations where it is important to keep costs of diagnosis low. We are currently evaluating cell immunity based assays for $\mathrm{TB}$ diagnosis in elephants. Further studies into TB transmission and surveillance using accurate, low cost and high throughput assays are also warranted. Such active disease surveillance in elephant range countries would help us to study the dynamic relationship between $\mathrm{TB}$ and elephant conservation.
Table 2. Sensitivity (DSe) and specificity (DSp) of the five serological tests used in the study.

\begin{tabular}{|c|c|c|c|}
\hline Test & w.r.t.** & DSe & DSp \\
\hline & & Median $(95 \% \mathrm{Cl})^{*}$ & Median $(95 \% \mathrm{Cl})^{*}$ \\
\hline \multirow[t]{5}{*}{ RT } & At least 1 antibody & $48.6(37.2,61.0)$ & $99.3(96.7,99.9)$ \\
\hline & ESAT6 & $44.6(33.9,56.4)$ & $95.2(90.4,98.2)$ \\
\hline & CFP10 & $46.5(36.0,58.1)$ & $93.0(87.8,96.6)$ \\
\hline & PE_PGRS11 & $79.8(66.7,90.8)$ & $98.5(93.6,99.9)$ \\
\hline & PE_PGRS17 & $84.5(75.2,91.3)$ & $89.6(79.4,98.4)$ \\
\hline \multirow[t]{5}{*}{ ESAT6 } & At least 1 antibody & $88.8(73.5,97.5)$ & $95.1(90.5,98.2)$ \\
\hline & ESAT6 & $91.3(74.8,99.5)$ & $95.2(90.4,98.2)$ \\
\hline & CFP10 & $77.0(61.9,88.5)$ & $88.9(83.2,93.6)$ \\
\hline & PE_PGRS11 & $85.0(71.7,94.0)$ & $75.9(68.2,82.3)$ \\
\hline & PE_PGRS17 & $80.3(66.7,90.2)$ & $65.7(57.8,73.1)$ \\
\hline \multirow[t]{5}{*}{ CFP10 } & At least 1 antibody & $88.8(73.5,97.5)$ & $95.3(90.8,98.2)$ \\
\hline & ESAT6 & $75.2(60.0,87.1)$ & $91.2(85.7,95.2)$ \\
\hline & CFP10 & $92.3(76.9,99.5)$ & $93.0(87.8,96.6)$ \\
\hline & PE_PGRS11 & $85.2(71.5,94.0)$ & $76.0(68.5,82.6)$ \\
\hline & PE_PGRS17 & $81.5(67.9,91.4)$ & $66.2(58.3,73.5)$ \\
\hline \multirow[t]{5}{*}{ PE_PGRS11 } & At least 1 antibody & $50.1(38.2,62.1)$ & $93.9(88.8,97.2)$ \\
\hline & ESAT6 & $47.1(35.8,58.7)$ & $91.5(85.9,95.4)$ \\
\hline & CFP10 & $49.1(38.0,60.2)$ & $89.4(83.5,93.7)$ \\
\hline & PE_PGRS11 & $97.6(88.6,99.8)$ & $98.5(93.6,99.9)$ \\
\hline & PE_PGRS17 & $87.1(77.6,93.6)$ & $81.4(73.4,88.1)$ \\
\hline \multirow[t]{5}{*}{ PE_PGRS17 } & At least 1 antibody & $44.4(32.5,57.6)$ & $91.6(85.5,96.0)$ \\
\hline & ESAT6 & $42.2(30.9,54.8)$ & $89.6(83.0,94.1)$ \\
\hline & CFP10 & $44.8(33.6,57.1)$ & $87.8(81.5,92.7)$ \\
\hline & PE_PGRS11 & $82.7(68.2,94.3)$ & $91.9(85.3,96.5)$ \\
\hline & PE_PGRS17 & $97.2(88.5,99.9)$ & $89.6(79.4,98.7)$ \\
\hline
\end{tabular}

The LCA model was used to calculate the DSe and DSp of each test w.r.t. the antibody it is designed to detect as also presence of at least one antibody. ${ }^{*}$ The Median estimate refers to the $50 \%$ quantile while the $2.5 \%$ and $97.5 \%$ quantiles define a $95 \%$ credible interval $(\mathrm{Cl}) .{ }^{* *}$ With respect to.

doi:10.1371/journal.pone.0049548.t002

\section{Materials and Methods}

\section{Study Population}

In the three southern Indian states of Kerala, Karnataka and Tamil Nadu, there are an estimated 1,000 Asian elephants in captivity. These animals, mostly caught from the wild but also born in captivity, are maintained under different ownership and management regimens. A project for captive elephant health assessment was undertaken by Asian Nature Conservation Foundation (Permit No.8-1/2002-PE, Project Elephant, Ministry of Environment and Forests, Government of India). Apart from photographic documentation of body condition index, wounds and injuries with special reference to eyes and feet, routine haematology, serum biochemistry, urinalysis and dung analysis were performed for individual elephants. In an attempt to provide better healthcare to these elephants, the results of each elephant's health evaluation was then handed over to the veterinarian incharge of the elephant. Following a convenience/opportunity sampling method, nearly 600 elephants were visited over a period of one year. A random sample of 179 serum samples collected from this heath survey was selected for this study. 
Table 3. Number of elephants (observed and predicted according to the latent class model) with each test result.

\begin{tabular}{|c|c|c|c|c|c|}
\hline $\begin{array}{l}\text { Binary } \\
\text { Input }\end{array}$ & $2.50 \%$ & $50 \%$ & $97.50 \%$ & Mean & Observed \\
\hline 00000 & 69 & 82 & 92 & 81 & 84 \\
\hline 00001 & 9 & 16 & 26 & 17 & 16 \\
\hline 00010 & 0 & 3 & 8 & 3 & 1 \\
\hline 00011 & 18 & 24 & 29 & 24 & 26 \\
\hline 00100 & 1 & 6 & 13 & 6 & 8 \\
\hline 00101 & 0 & 1 & 4 & 1 & 1 \\
\hline 00110 & 0 & 0 & 2 & 0 & 1 \\
\hline 00111 & 0 & 2 & 6 & 2 & 0 \\
\hline 01000 & 0 & 4 & 11 & 4 & 4 \\
\hline 01001 & 0 & 1 & 3 & 1 & 1 \\
\hline 01010 & 0 & 0 & 2 & 0 & 0 \\
\hline 01011 & 0 & 1 & 5 & 2 & 2 \\
\hline 01100 & 0 & 0 & 2 & 0 & 0 \\
\hline 01101 & 0 & 0 & 1 & 0 & 0 \\
\hline 01110 & 0 & 0 & 2 & 0 & 0 \\
\hline 01111 & 0 & 2 & 8 & 3 & 2 \\
\hline 10000 & 0 & 0 & 4 & 1 & 0 \\
\hline 10001 & 0 & 0 & 1 & 0 & 0 \\
\hline 10010 & 0 & 0 & 1 & 0 & 0 \\
\hline 10011 & 0 & 1 & 3 & 1 & 1 \\
\hline 10100 & 0 & 0 & 2 & 0 & 0 \\
\hline 10101 & 0 & 0 & 2 & 0 & 0 \\
\hline 10110 & 0 & 0 & 2 & 1 & 0 \\
\hline 10111 & 1 & 4 & 8 & 4 & 4 \\
\hline 11000 & 0 & 0 & 2 & 0 & 0 \\
\hline 11001 & 0 & 0 & 2 & 0 & 0 \\
\hline 11010 & 0 & 1 & 3 & 1 & 1 \\
\hline 11011 & 0 & 3 & 8 & 3 & 2 \\
\hline 11100 & 0 & 1 & 2 & 1 & 1 \\
\hline 11101 & 0 & 0 & 3 & 1 & 0 \\
\hline 11110 & 0 & 2 & 5 & 2 & 2 \\
\hline 11111 & 12 & 19 & 25 & 19 & 22 \\
\hline
\end{tabular}

Evaluating the fit of the model by comparing the observed and expected number of elephants with different combinations of tests to see if the assumptions of the substantive model in Figure 2 were satisfied. $50 \%$ refers to the median estimate while the $2.5 \%$ and $97.5 \%$ quantiles define a $95 \%$ credible interval $(\mathrm{Cl})$.

doi:10.1371/journal.pone.0049548.t003

\section{Materials}

Unless otherwise specified all materials used in this study were purchased from Sigma-Aldrich, St. Louis, USA. Vacutainer needles (No. 301747), Vacutainer tubes (No. 367820), PANTA ${ }^{\mathrm{TM}}$ Supplement, BACTEC ${ }^{\text {TM }}$ 12B Mycobacteria Culture Vials and BBL $^{\text {TM }}$ Mycobactose Lowenstein-Jensen Medium were procured from BD (Franklin Lakes, NJ, USA). GammaBind G, Type 2 affinity matrix was bought from GE Healthcare Bio-Sciences (Uppsala, Sweden), Ni-nitrilotriacetic acid (Ni-NTA) columns from Qiagen (Valencia, CA, USA), Nunc-Immuno Plates (No 44204) from NUNC A/S (Roskilde, Denmark) and polyvinylidene difluoride membranes (PVDF) from Millipore (Bedford, MA, USA). ECL detection system was bought from Perkin-Elmer (MA,
USA) and 3,3',5,5'-tetramethylbenzidine (TMB) and horse radish peroxidase (HRP)-labeling kit from Bangalore Genei (Bangalore, Karnataka, India).

\section{Collection of Serum and Screening with RT}

Blood was collected by venipuncture of the middle auricular vein, allowed to clot at room temperature and serum separated within three hours of collection by centrifugation at $500 \mathrm{~g}$ for 10 minutes. Each serum was screened with RT and stored at $-70^{\circ} \mathrm{C}$ until further testing. The RT (http://www.chembio.com/ animaltest $4 . h$ tml) is a point-of-care lateral flow serological test licensed by the USDA in 2007. Greenwald et al. [22] reported a DSp of $95.2 \%$ (95\% CI, 90.1 to 97.9$)$ and DSe of $100 \%$ (95\% CI, $84.0 \%-100 \%)$ for the RT.

\section{Trunk-wash Culture for Isolation of M. tuberculosis}

The procedure for trunk wash collection as described in the Guidelines for the Control of Tuberculosis in Elephants, 2008 [15] was modified [69]. Trunk wash specimens from select RT positive elephants were tested for M. tuberculosis culture [15]. Briefly, $0.5 \mathrm{ml}$ of sample supplemented with Erythromysin $(32 \mu \mathrm{g} / \mathrm{ml})$ and PANTA $^{\text {TM }}$ Supplement was inoculated into BACTEC ${ }^{\mathrm{TM}} 12 \mathrm{~B}$ vials and $\mathrm{BBL}^{\mathrm{TM}}$ Mycobactose Lowenstein-Jensen Medium and grown at $37 \mathrm{C}$ and $10 \% \mathrm{CO}_{2}$ for 8 weeks; this was followed by a niacin-nitrate reduction test for confirming $M$. tuberculosis. Antemortem serum collected from one elephant, which showed nodules in the lung tissue during post-mortem examination and from which $M$. tuberculosis was cultured on Lowenstein-Jensen medium, was used as positive control in the serological assays.

\section{Expression and Purification of Recombinant ESAT6, CFP10, PE-PGRS17 AND PE-PGRS11 Proteins}

M. tuberculosis $\mathrm{H} 37 \mathrm{Rv}$ antigens EsxA-6 kDa early secretory antigenic target (ESAT-6) (Rv3875); EsxB-10 kDa culture filtrate antigen (CFP10) (Rv3874); PE_PGRS17 (Rv0978c) and PE_PGRS11 (Rv0754) were used as the major antigenic determinants in this study. Recombinant expression vectors for Rv3875, Rv3874, Rv0978c and Rv0754 antigens were obtained from Colorado State University, TB Vaccine Testing and Research Materials Contract (http://www.cvmbs.colostate.edu/ $\mathrm{mip} / \mathrm{tb} /$ recombinant.htm) and expressed as described previously [70]. Briefly, for the expression of His-tagged recombinant antigens, Escherichia coli BL21(DE3) cells carrying recombinant plasmids were induced with isopropyl-b-D-thiogalactopyranoside, and the proteins purified under native conditions (ESAT6 and CFP10) and denaturing conditions (PE_PGRS17 and PE_PGRS1 1) using Ni-NTA columns. In-gel digestions of proteins for matrix-assisted laser desorption/ionization mass spectrometry was carried out for identification.

\section{Raising Rabbit Anti-Asian Elephant IgG-horse Radish Peroxidase (HRP)}

Asian elephant IgG was separated from sera [71] and rabbit anti-Asian elephant IgG raised as per [59] and [60] with modifications. Briefly, New Zealand white rabbits were injected subcutaneously at multiple sites with $1 \mathrm{mg}$ of purified Asian elephant IgG emulsified in equal volume of Freund's complete adjuvant followed by a second dose of $500 \mu \mathrm{g}$ Asian elephant IgG emulsified in Freund's incomplete adjuvant. Antibody titres in sera were determined two weeks post final immunization by ELISA. The rabbit anti-Asian elephant IgG was coupled to HRP and its reactivity to elephant IgG was checked. All animal experiments were approved by the Institutional Ethics Committee for Animal 
Experimentation and Institutional Biosafety Committee, Indian Institute of Science, Bangalore.

\section{ELISA}

Careful checker board titration for optimum protein concentration, elephant sera dilution, rabbit anti-Asian elephant IgGHRP was carried out for each individual ELISA. All protein dilutions were made in 1X PBS $(137 \mathrm{mM} \mathrm{NaCl}$; $2.7 \mathrm{mM} \mathrm{KCl}$; $4.3 \mathrm{mM} \mathrm{Na} 2 \mathrm{HPO} 4 ; 1.47 \mathrm{mM} \mathrm{KH} 2 \mathrm{PO} 4, \mathrm{pH} 7.4$ ). 1X PBST (1X PBS with $0.05 \%$ tween-20) was used as wash buffer and $3 \%$ BSA in PBST as blocking buffer. Elephant sera, rabbit anti-Asian elephant IgG-HRP were each diluted in blocking buffer and $100 \mu \mathrm{l}$ added per well. ESAT-6 $(1 \mu \mathrm{g} / \mathrm{ml})$, CFP10 $(0.5 \mu \mathrm{g} / \mathrm{ml})$, PE_PGRS17 and PE_PGRS11 $(0.25 \mu \mathrm{g} / \mathrm{ml}$ each $)$ were coated overnight $(\mathrm{o} / \mathrm{n})$ at $4^{\circ} \mathrm{C}$ into ELISA plates and then washed thrice. Blocking for 1 hour was followed by addition of elephant sera $(1: 200)$ and incubation $\mathrm{o} / \mathrm{n}$ at $4^{\circ} \mathrm{C}$. After washing, rabbit antiAsian elephant IgG-HRP (1:3000) was added and the plate incubated $\mathrm{o} / \mathrm{n}$ at $4{ }^{\circ} \mathrm{C}$. Tetramethylbenzidine was used as chromogenic substrate and the absorbance was read at $450 \mathrm{~nm}$ using an ELISA reader (Molecular Devices, Sunnyvale, CA, USA).

\section{Immunoblot Analysis}

Ten $\mu \mathrm{g}$ of purified protein was subjected to $12 \%$ SDS-PAGE (Laemmeli) or 10\% Tricine SDS-PAGE; transferred to PVDF and stained with Ponceau to check for loading control. The PVDF was cut into strips, blocked with $5 \%$ nonfat dried milk and each strip probed with individual elephant sera overnight at $4{ }^{\circ} \mathrm{C}$, probed with rabbit anti-Asian elephant IgG-HRP and the blot visualized with the ECL detection system.

\section{Statistical Analysis}

To adjust for the imperfect nature of the gold-standard reference, our test validation entailed i) determining an optimal cut-off for each ELISA using a mixture model for continuous data ii) using LCA to estimate the DSe and DSp of the five dichotomous tests. The first step was carried out using the mixdist library in the $\mathrm{R}$ software package [72] assuming that the observed continuous data on each test arises from a mixture of two Weibull distributions among the antibody positive and antibody negative elephants. The point of intersection of the two density functions was chosen as the optimal cut-off.

\section{References}

1. Jones KE, Patel NG, Levy MA, Storeygard A, Balk D, et al. (2008) Global trends in emerging infectious diseases. Nature 451: 990-993.

2. Aguirre AA, Gomez A (2009) Essential veterinary education in conservation medicine and ecosystem health: a global perspective. Rev Sci Tech 28: 597-603.

3. Choudhury A, Lahiri Choudhury DK, Desai A, Duckworth JW, Easa PS, et al. (2010) Elephas maximus. IUCN 2010. IUCN Red List of Threatened Species Version 20102, 2010.

4. Fernando P, J P (2011) Range-wide Status of Asian Elephants. Gajah 35: 15-29.

5. Bist SS (2006) Elephant conservation in India - an overview. Gajah 25: 27-37.

6. Michalak K, Austin C, Diesel S, Bacon MJ, Zimmerman P, et al. (1998) Mycobacterium tuberculosis infection as a zoonotic disease: transmission between humans and elephants. Emerg Infect Dis 4: 283-287.

7. Davis M (2001) Mycobacterium tuberculosis risk for elephant handlers and veterinarians. Appl Occup Environ Hyg 16: 350-353.

8. Montali RJ, Mikota SK, Cheng LI (2001) Mycobacterium tuberculosis in zoo and wildlife species. Rev Sci Tech 20: 291-303.

9. Oh P, Granich R, Scott J, Sun B, Joseph M, et al. (2002) Human exposure following Mycobacterium tuberculosis infection of multiple animal species in a Metropolitan Zoo. Emerg Infect Dis 8: 1290-1293.

10. Murphree R, Warkentin JV, Dunn JR, Schaffner W, Jones TF (2011) Elephantto-human transmission of tuberculosis, 2009. Emerg Infect Dis 17: 366-371.

11. Mikota SK, Maslow JN (2011) Tuberculosis at the human-animal interface: an emerging disease of elephants. Tuberculosis (Edinb) 91: 208-211.
The LCA was carried out using the lcmr library in R software package [43]. This package uses a Bayesian approach to estimate the parameters of interest. We used non-informative prior distributions over all parameters so as to let the data dominate the analysis. The LCA model (Figure 2) assumed that each of the ELISA tests was measuring a different latent variable (i.e. true presence of the antibody) which was present if $M$. tuberculosis infection was present. Thus, this model adjusts for the correlation that may arise between tests within the groups of elephants that are $M$. tuberculosis infection positive or negative. The RT test was assumed to measure the presence of both ESAT-6 and CFP10 antibodies while each of the four ELISA was assumed to measure the presence of the corresponding antibody. The resulting model had 16 latent classes corresponding to different combinations of the antibodies (see supplementary document File S1). The fit of the model was evaluated by comparing the observed and expected number of elephants with different combinations of tests and a posterior predictive check for conditional dependence (Table 3). The predictive values of each test combination were examined to see if the assumptions of the substantive model in Figure 2 were satisfied.

\section{Supporting Information}

\section{File S1 Algorithm for LGA model. (DOC)}

\section{Acknowledgments}

We gratefully acknowledge the support extended by Director, Project Elephant, Ministry of Environment and Forests, Government of India, and the state forest departments of Karnataka, Tamil Nadu and Kerala. We thank the TB Vaccine Testing and Research Materials Contract at Colorado State University for the gift of recombinant expression vectors used in this study. We acknowledge help by B.C. Chittiappa and other field veterinarians. We are grateful to Thomas Mathew, Kavita Iswaran, Karpagam Chelliah, Devram Sampat Ghorpade, Sahana Holla, Kushagra Bansal, Nisha Kapoor and Yeddula Narayana for their contributions to this study.

\section{Author Contributions}

Conceived and designed the experiments: SVK DA KNB RS. Performed the experiments: SVK DA. Analyzed the data: ND SVK. Contributed reagents/materials/analysis tools: RS JVC ND KNB. Wrote the paper: SVK ND DA RS KNB

12. Keilty RA (1915) A Study of the Cultivation of the Tubercle Bacillus Directly from the Sputum by the Method of Petroff. J Exp Med 22: 612-614.

13. Chu LS (1955) Rapid method for cultivation of acid-fast bacilli. Science 122: 1189-1190

14. Rachow A, Zumla A, Heinrich N, Rojas-Ponce G, Mtafya B, et al. (2011) Rapid and accurate detection of Mycobacterium tuberculosis in sputum samples by Cepheid Xpert MTB/RIF assay-a clinical validation study. PLoS One 6: e20458.

15. The National Tuberculosis Working Group for Zoo and Wildlife Species (2008) USDA. Guidelines for the control of tuberculosis in elephants, 2008. Available: http://www.aphis.usda.gov/animal_welfare/downloads/elephant/elephant_tb. pdf Accessed 2009 Jun 10

16. Moller T, Roken B, Petersson L, Vitaud C, Lyashchenko K (2005) Preliminary results of a new serological test for detection of TB-infection (Mycobacterium tuberculosis) in elephants (Elephas maximus and Loxodonta africanum) - Swedish Case studies. VerhberErkrgZootiere 42: 173-181.

17. Mikota SK, Peddie L, Peddie J, Isaza R, Dunker F, et al. (2001) Epidemiology and diagnosis of Mycobacterium tuberculosis in captive Asian elephants (Elephas maximus). J Zoo Wildl Med 32: 1-16.

18. Lewerin SS, Olsson SL, Eld K, Roken B, Ghebremichael S, et al. (2005) Outbreak of Mycobacterium tuberculosis infection among captive Asian elephants in a Swedish zoo. Vet Rec 156: 171-175.

19. Payeur JB, Jarnagin JL, Marquardt JG, Whipple DL (2002) Mycobacterial isolations in captive elephants in the United States. Ann N Y Acad Sci 969: 256258. 
20. Larsen RS, Salman MD, Mikota SK, Isaza R, Montali RJ, et al. (2000) Evaluation of a multiple-antigen enzyme-linked immunosorbent assay for detection of Mycobacterium tuberculosis infection in captive elephants. J Zoo Wildl Med 31: 291-302.

21. Lyashchenko KP, Greenwald R, Esfandiari J, Olsen JH, Ball R, et al. (2006) Tuberculosis in elephants: antibody responses to defined antigens of Mycobacterium tuberculosis, potential for early diagnosis, and monitoring of treatment. Clin Vaccine Immunol 13: 722-732.

22. Greenwald R, Lyashchenko O, Esfandiari J, Miller M, Mikota S, et al. (2009) Highly accurate antibody assays for early and rapid detection of tuberculosis in African and Asian elephants. Clin Vaccine Immunol 16: 605-612.

23. Lyashchenko KP, Greenwald R, Esfandiari J, Mikota S, Miller M, et al. (2012) Field Application of Serodiagnostics to Identify Elephants with Tuberculosis prior to Case Confirmation by Culture. Clin Vaccine Immunol.

24. Harboe M, Oettinger T, Wiker HG, Rosenkrands I, Andersen P (1996) Evidence for occurrence of the ESAT-6 protein in Mycobacterium tuberculosis and virulent Mycobacterium bovis and for its absence in Mycobacterium bovis BCG. Infect Immun 64: 16-22.

25. Skjot RL, Oettinger T, Rosenkrands I, Ravn P, Brock I, et al. (2000) Comparative evaluation of low-molecular-mass proteins from Mycobacterium tuberculosis identifies members of the ESAT- 6 family as immunodominant T-cell antigens. Infect Immun 68: 214-220.

26. Cole ST, Brosch R, Parkhill J, Garnier T, Churcher C, et al. (1998) Deciphering the biology of Mycobacterium tuberculosis from the complete genome sequence. Nature 393: 537-544.

27. Chaturvedi R, Bansal K, Narayana Y, Kapoor N, Sukumar N, et al. (2010) The multifunctional PE_PGRS11 protein from Mycobacterium tuberculosis plays a role in regulating resistance to oxidative stress. J Biol Chem 285: 30389-30403.

28. Bansal K, Elluru SR, Narayana Y, Chaturvedi R, Patil SA, et al. (2010) PE_PGRS antigens of Mycobacterium tuberculosis induce maturation and activation of human dendritic cells. J Immunol 184: 3495-3504.

29. Alonzo TA, Pepe MS (1999) Using a combination of reference tests to assess the accuracy of a new diagnostic test. Stat Med 18: 2987-3003.

30. World Organization for Animal Health (OIE) (2010) Principles of validation of diagnostic assays for infectious diseases. Manual of Diagnostic Tests and Vaccines for Terrestrial Animals OIE, Paris, France: 1-18.

31. TDR Diagnostics Evaluation Expert Panel, Banoo S, Bell D, Bossuyt P, Herring A, et al. (2010) Evaluation of diagnostic tests for infectious diseases: general principles. Nat Rev Microbiol 8: S17-29.

32. Rindskopf D, Rindskopf W (1986) The value of latent class analysis in medical diagnosis. Stat Med 5: 21-27.

33. Qu Y, Tan M, Kutner MH (1996) Random effects models in latent class analysis for evaluating accuracy of diagnostic tests. Biometrics 52: 797-810.

34. Pepe MS, Janes H (2007) Insights into latent class analysis of diagnostic test performance. Biostatistics 8: 474-484.

35. Boelaert M, Aoun K, Liinev J, Goetghebeur E, Van der Stuyft P (1999) The potential of latent class analysis in diagnostic test validation for canine Leishmania infantum infection. Epidemiol Infect 123: 499-506.

36. Frossling J, Bonnett B, Lindberg A, Bjorkman C (2003) Validation of a Neospora caninum iscom ELISA without a gold standard. Prev Vet Med 57: 141-153.

37. Rose N, Boutrouille A, Fablet C, Madec F, Eloit M, et al. (2010) The use of Bayesian methods for evaluating the performance of a virus-like particles-based ELISA for serology of hepatitis $\mathrm{E}$ virus infection in swine. J Virol Methods 163: 329-335.

38. Clegg TA, Duignan A, Whelan C, Gormley E, Good M, et al. (2011) Using latent class analysis to estimate the test characteristics of the gamma-interferon test, the single intradermal comparative tuberculin test and a multiplex immunoassay under Irish conditions. Vet Microbiol 151: 68-76.

39. Morton JM, McCoy RJ, Kann RK, Gardner IA, Meers J (2012) Validation of real-time polymerase chain reaction tests for diagnosing feline immunodeficiency virus infection in domestic cats using Bayesian latent class models. Prev Vet Med 104: $136-148$

40. Greiner M, Gardner IA (2000) Epidemiologic issues in the validation of veterinary diagnostic tests. Prev Vet Med 45: 3-22.

41. More SJ, Cameron AR, Greiner M, Clifton-Hadley RS, Rodeia SC, et al. (2009) Defining output-based standards to achieve and maintain tuberculosis freedom in farmed deer, with reference to member states of the European Union. Prev Vet Med 90: 254-267.

42. Pai M, Dendukuri N, Wang L, Joshi R, Kalantri S, et al. (2008) Improving the estimation of tuberculosis infection prevalence using T-cell-based assay and mixture models. Int J Tuberc Lung Dis 12: 895-902.

43. Dendukuri N, Hadgu A, Wang L (2009) Modeling conditional dependence between diagnostic tests: a multiple latent variable model. Stat Med 28: 441461.

44. Kunnath-Velayudhan S, Salamon H, Wang HY, Davidow AL, Molina DM, et al. (2010) Dynamic antibody responses to the Mycobacterium tuberculosis proteome. Proc Natl Acad Sci U S A 107: 14703-14708.

45. Davidow A, Kanaujia GV, Shi L, Kaviar J, Guo X, et al. (2005) Antibody profiles characteristic of Mycobacterium tuberculosis infection state. Infect Immun 73: 6846-6851.
46. Kunnath-Velayudhan S, Gennaro ML (2011) Immunodiagnosis of tuberculosis: a dynamic view of biomarker discovery. Clin Microbiol Rev 24: 792-805.

47. Steingart KR, Dendukuri N, Henry M, Schiller I, Nahid P, et al. (2009) Performance of purified antigens for serodiagnosis of pulmonary tuberculosis: a meta-analysis. Clin Vaccine Immunol 16: 260-276.

48. Diel R, Loddenkemper R, Nienhaus A (2010) Evidence-based comparison of commercial interferon-gamma release assays for detecting active TB: a metaanalysis. Chest 137: 952-968.

49. Sester M, Sotgiu G, Lange C, Giehl C, Girardi E, et al. (2011) Interferongamma release assays for the diagnosis of active tuberculosis: a systematic review and meta-analysis. Eur Respir J 37: 100-111.

50. Steingart KR, Flores LL, Dendukuri N, Schiller I, Laal S, et al. (2011) Commercial serological tests for the diagnosis of active pulmonary and extrapulmonary tuberculosis: an updated systematic review and meta-analysis. PLoS Med 8: e1001062.

51. Arloing S (1898) Agglutination de bacille de la tuberculose vraie. Les Comptes Rendus de l'Academie des Sciences 126: 1398-1400.

52. Narayana Y, Joshi B, Katoch VM, Mishra KC, Balaji KN (2007) Differential Bcell responses are induced by Mycobacterium tuberculosis PE antigens Rv1169c, Rv0978c, and Rv1818c. Clin Vaccine Immunol 14: 1334-1341.

53. Espitia C, Laclette JP, Mondragon-Palomino M, Amador A, Campuzano J, et al. (1999) The PE-PGRS glycine-rich proteins of Mycobacterium tuberculosis: a new family of fibronectin-binding proteins? Microbiology 145 (Pt 12): 3487-3495.

54. Singh KK, Dong Y, Patibandla SA, McMurray DN, Arora VK, et al. (2005) Immunogenicity of the Mycobacterium tuberculosis PPE55 (Rv3347c) protein during incipient and clinical tuberculosis. Infect Immun 73: 5004-5014.

55. Aagaard C, Govaerts M, Meikle V, Vallecillo AJ, Gutierrez-Pabello JA, et al. (2006) Optimizing antigen cocktails for detection of Mycobacterium bovis in herds with different prevalences of bovine tuberculosis: ESAT6-CFP10 mixture shows optimal sensitivity and specificity. J Clin Microbiol 44: 4326-4335.

56. Ameni G, Aseffa A, Engers H, Young D, Hewinson G, et al. (2006) Cattle husbandry in Ethiopia is a predominant factor affecting the pathology of bovine tuberculosis and gamma interferon responses to mycobacterial antigens. Clin Vaccine Immunol 13: 1030-1036.

57. Lafferty K (2002) Good medicine for conservation biology: The intersection of epidemiology and conservation theory. Conservation Biology 16: 593-604.

58. Michel AL, Venter L, Espie IW, Coetzee ML (2003) Mycobacterium tuberculosis infections in eight species at the National Zoological Gardens of South Africa, 1991-2001. J Zoo Wildl Med 34: 364-370.

59. Kania SA, Richman LK, Kennedy MA, Montali RJ, Potgieter LND (1997) The isolation, Detection, and Cross-Reactivity of Asian Elephant IgG for the Development of Serological Diagnostic Tests. Veterinary Allergy Clinical Immunology 5: 125-128.

60. Kelly PJ, Carter SD, Azwai SM, Cadman HF (1998) Isolation and characterisation of immunoglobulin $\mathrm{g}$ and $\mathrm{IgG}$ subclasses of the African elephant (Loxodonta africana). Comp Immunol Microbiol Infect Dis 21: 65-73.

61. Guo Y, Bao Y, Wang H, Hu X, Zhao Z, et al. (2011) A preliminary analysis of the immunoglobulin genes in the African elephant (Loxodonta africana). PLoS One 6: e16889.

62. Archie E, Henry T, Maldonado J, Moss C, Poole J, et al. (2010) Major histocompatibility complex variation and evolution at a single, expressed DQA locus in two genera of elephants. Immunogenetics 62: 85-100.

63. Mushi EZ, Hill FWG, Dawe P, Riess R (1990) Antibodies to bluetongue and African horse sickness viruses in the sera of elephants in Zimbabwe. Bulletin of Animal Health and Production in Africa 38: 475.

64. Davies F, Otieno S (1977) Elephants and zebras as possible reservoir hosts for African horse sickness virus. Veterinary Record 100: 291-292.

65. Clark HW, Laughlin DG, Bailey JS, Brown TM (1980) Mycoplasma Species and Arthritis in Captive Elephants. The Journal of Zoo Animal Medicine 11: 3-15.

66. Rajan A, Vikram-Reddy M, Sulochana S, Valsala K (1980) Demonstration of T lymphocyte distribution in the peripheral blood of Indian elephant (Elephas maximus) using acid Alpha Napthyl Acetate Esterase activity as a T cell marker.. African Journal of Clinical Experimental Immunology 2: 357-362

67. Looringh van Beeck F, Reinink P, Hermsen R, Zajonc D, Laven M, et al. (2009) Functional CDld and/or NKT cell invariant chain transcript in horse, pig, African elephant and guinea pig, but not in ruminants. Molecular Immunology 46: 1424-1431.

68. Khan IH, Ravindran R, Yee J, Ziman M, Lewinsohn DM, et al. (2008) Profiling antibodies to Mycobacterium tuberculosis by multiplex microbead suspension arrays for serodiagnosis of tuberculosis. Clin Vaccine Immunol 15: 433-438.

69. Abraham D, Davis J (2008) Revised Trunk was collection procedure for captive elephants in a range country setting. Gajah 28: 46-47.

70. A SK, Bansal K, Holla S, Verma-Kumar S, Sharma P, et al. (2012) ESAT-6 induced COX-2 expression involves coordinated interplay between PI3K and MAPK signaling. Mol Immunol 49: 655-663.

71. Manual AAL (1988) Protocols for Immunaffinity purification. In: Harlow E, Lane D, editors. Cold Spring Harbor Laboratory Press: Cold Spring Harbor, New York. 519-554

72. Macdonald PDM, Du J (2010) Mixdist: Finite Mixture Distribution Models. R package version 0.5-3. 2010.Available: http://cran.r-project.org/web/ packages/mixdist/index.html. Accessed 2011 Sep 21 